\title{
ARCHIVES OF PHOTOMETRIC DATA
}

\author{
J.-C. Mermilliod \\ Institute of Astronomy, Lausanne University \\ 1290 Chavannes-des-Bois \\ Switzerland
}

\section{INTRODUCTION}

The process of archiving photometric data and compiling catalogues is in principle easy to solve, but, in practice, does not appear so simple. Data retrieval may become difficult and inefficient if sufficient care is not taken to solve the general problems which are encoutered: (i) the lack of definition of fundamental identificators; (ii) the lack of coordinates for thousands of faint stars; (iii) the innacuracy of the identification and description of the components observed in double stars or multiple systems: A, B, C, $\mathrm{AB}$, or $\mathrm{BC}$; (iv) the fact that the number of observations is often not published.

The first item (i) means that a lot of different identificators are used and that a long job has to be done to prepare a homogeneous system of stellar identifications. The second one (ii) implies that still too many stars are identified only on charts and are lost when catalogues are searched by coordinates.

Generally speaking, we need what I shall call active archives. This means two different kinds of action: (a) to collect the data and check their quality by intercomparison of the various sources. (b) to develop interaction with the observers to improve the completeness of interesting samples of stars.

This seems the only way to be sure of having now and in the future reliable and complete data, which will still retain some interest for the next generations of astronomers.

\section{STATUS OF THE ARCHIVES}

The status of the archives of photometric data is different for various kinds of objects and I have reviewed: (1) the photoelectric data of galactic stars; (2) the data for stars in open clusters ; (3) in globular clusters; (4) stars in external galaxies ; and (5) CCD data of field stars .

\subsection{Photoelectric data for galactic stars}

They have been collected since 1970 at the Institute of Astronomy of Lausanne University and are now fairly complete. The collection contains data for some 166000 stars in 78 photometric systems. A description has been published by Hauck et al (1990). New 
mean values for the UBV systems have been computed and the publication of a catalogue containing not only the UBV mean data but also cross-identifications, coordinates, spectral types and references by Springer are in preparation. Infrared data have been compiled extensively by Gezari et al (1984).

No equivalent compilation exists for photographic data.

\subsection{Stars in open clusters}

Most problems raised by open clusters (i.e. the numerous numbering systems used in each cluster, the lack of coordinates) have been largely solved by the development of a specialised database (Mermilliod 1988), which contains extensive collections of photometric data (UBV photoelectric: 28940 measures, photographic: 82000 measures, CCD: 14000 measures), as well as most other available information (spectral types, radial velocity, rotational velocity, membership probability, positions, and so on).

\subsection{Stars in globular clusters}

Philip, Cullen and White (1976) had collected most photographic data for stars in globular clusters during the preparation of their atlas of colour-magnitude diagrams. Since that time numerous photographic and CCD studies have been published, but I am not aware of any group collecting these new data systematically. There is of course a large problem of cross-identifications between the various investigations of the same clusters. This domain is in need of a long-term global solution and a specific database would be a good one.

The same remarks are also valid for LMC and SMC clusters.

\subsection{Stars in external galaxies}

To my knowledge, nothing has been undertaken in the developing field of photometric observations of stars in external galaxies. The kind of identification used so far does not facilitate the archiving task. One should think of a systematic way for identifying stars in external galaxies.

\subsection{CCD observations}

Another talk is devoted to the problem of CCD data. The general interest of keeping all the data is not evident due to the small fields involved, except for calibrating photographic plates. I would propose that a list of existing fields published, with indication of central position, type of filters, limiting magnitudes, number of stars, should be made available in the Data Centers and that a copy of the reduced data be deposited there with public access. Stars could be designated by the field number, attributed by the Data Centers, and the authors' star numbers.

\section{REFERENCES}

Gezari D.Y, Schmitz M., Mead J.M., 1984, NASA Ref. Publ. 1118

Hauck B., Nitschelm C., Mermilliod M., Mermilliod J.-C., 1990, A\&AS 85,989-998

Mermilliod J.-C., 1988, Bull. Inform. CDS 35, 77-91

Philip A.G.D., Cullen M.F., White R.E., (1976) Dudley Obs. report no 11 